\title{
Management of patients with Graves' orbitopathy: initial assessment, management outside specialised centres and referral pathways
}

\author{
Authors: Petros Perros, ${ }^{\mathrm{A}}$ Colin M Dayan, ${ }^{\mathrm{B}}$ A Jane Dickinson, ${ }^{\mathrm{C}}$ Daniel Ezra, ${ }^{\mathrm{D}}$ Stephanie Estcourt, ${ }^{\mathrm{E}}$ Peter Foley, ${ }^{\mathrm{F}}$ Janis
} Hickey, ${ }^{\mathrm{G}}$ John $\mathrm{H}$ Lazarus, ${ }^{\mathrm{H}}$ Caroline J MacEwen, ${ }^{\mathrm{I}}$ Julie McLaren, ${ }^{\mathrm{J}}$ Geoffrey E Rose, ${ }^{\mathrm{K}}{ }^{\text {Jimmy }}$ Uddin ${ }^{\mathrm{L}}$ and Bijay Vaidya ${ }^{\mathrm{M}}$

\begin{abstract}
Graves' orbitopathy (GO) is uncommon, but responsible for considerable morbidity. A coordinated approach between healthcare professionals is required in order to meet the needs of patients. Early diagnosis can be achieved by a simple clinical assessment. Low-cost effective interventions can be initiated by generalists, which may improve outcomes. Moderateto-severe GO should be referred to specialised centres. Recommendations for clinical diagnosis, initial management and referral pathways are highlighted.
\end{abstract}

KEYWORDS: Graves' orbitopathy, thyroid eye disease, guidance, referral pathways.

\section{Introduction}

Graves' orbitopathy (GO) is an uncommon, disfiguring and disabling autoimmune condition and more than $90 \%$ of cases occur in patients presenting with hyperthyroidism due to Graves' disease. If untreated, GO is characterised by an initial phase of gradual deterioration lasting 6-12 months,

Authors: Aconsultant endocrinologist, Newcastle upon Tyne Hospitals NHS Foundation Trust, Newcastle upon Tyne, UK; Bprofessor of clinical diabetes and metabolism director, Institute of Molecular and Experimental Medicine, Cardiff University, Cardiff, UK; C retired consultant ophthalmologist, Newcastle upon Tyne Hospitals NHS Foundation Trust, Newcastle upon Tyne, UK; ${ }^{D}$ consultant ophthalmologist, NIHR Biomedical Research Centre for Ophthalmology, Moorfields Eye Hospital, London, UK; Eresearch nurse specialist - team lead, Royal Devon and Exeter Hospital, Exeter, UK; FTEAMeD patient member (British Thyroid Foundation), Harrogate, UK; ${ }^{G}$ director, British Thyroid Foundation, Harrogate, UK; ${ }^{H}$ emeritus professor clinical endocrinology, Cardiff University,

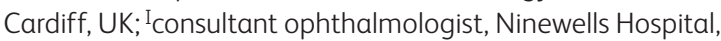
Dundee, UK; 'TEAMeD patient member - representing Thyroid

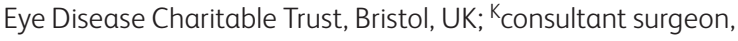

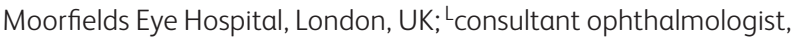
Moorfields Eye Hospital, London, UK; ${ }^{M}$ Consultant endocrinologist, Royal Devon and Exeter Hospital, Exeter, UK and then slow improvement lasting for 2-3 years. Optimal treatment improves quality of life. ${ }^{1} \mathrm{GO}$ is often misdiagnosed as conjunctivitis or allergy. ${ }^{2}$ Delays in diagnosis and treatment compromise outcomes. Treatments that can improve visual function and quality of life are often not accessible to patients. ${ }^{3}$

\section{Scope and purpose}

The Amsterdam Declaration on Graves' orbitopathy ${ }^{4}$ is an international initiative, which highlighted areas requiring improvement in the care of patients with GO, and set targets for earlier diagnosis, referral and treatment. The authors of this guidance are members of the Thyroid Eye Disease Amsterdam Declaration Implementation Group UK (TEAMeD) ${ }^{5}$, which was formed in 2010 to promote the objectives of the Amsterdam Declaration in the UK.

This guidance aims to promote early recognition and improve management of adult patients with GO and identify those who may benefit from referral to specialised centres.

\section{Recommendations}

Levels of evidence were graded according to SIGN $50 .^{6}$ These are indicated in each case where the evidence base is stronger than guideline development group experience and opinion alone. Particular good practice points are emphasised thus $\square$. Relevant supplementary source data (S1-5) are referred to and available online at http://www.btf-thyroid.org/index. php/campaigns/teamed (S1, common associated symptoms and signs of GO; 2 , algorithmic diagnostic criteria for GO; S3, activity and severity assessments for patients with GO; S4, DiaGO (diagnosis of GO): GO clinical assessment tool; S5, early symptoms of GO: information for patients.)

The National Institute of Health and Care Excellence (NICE) has accredited the process used by the Royal College of Physicians to produce the concise clinical guidelines published in Clinical Medicine with effect February 2010 to March 2018 (abstracted guidance) and July 2013 to July 2018 (de novo guidance). More information on accreditation can be viewed at: www.nice.org.uk/about/what-we-do/accreditation. 


\section{Box 1. Clinical diagnosis of GO.}

Early symptoms of patients with undiagnosed GO

$>$ Redness in the eyes or lids

Swelling or feeling of fullness in one or both upper eyelids

$>$ Bags under the eyes

Eyes seem to be too wide open

$>$ Pain in or behind the eyes

\section{Discriminating points in favour of a diagnosis of $\mathrm{GO}$}

$>$ Known or suspected thyroid dysfunction, particularly if recent onset (within 18 months)

$>$ Symptoms unrelieved by topical antibiotics

Eyelids abnormally wide (upper or lower eyelid retraction)

$>$ New 'bags' in eyelids upper or lower

> Change in appearance of eyes and eyelids particularly eyeball protrusion with eyelid swelling or widened lid aperture

> Presence of other signs frequently associated with GO: double vision, inability to fully close the eyes

\section{Discriminating points against a diagnosis of $\mathrm{GO}$}

$>$ Predominantly itchy eyes or eyelids

Mucoid or purulent discharge

Other family members newly affected simultaneously

Drooping upper eyelids

> Unilateral symptoms or signs, although $\mathrm{GO}$ is often asymmetrical and unilateral in $15 \%$ of cases.

GO = Graves' orbitopathy

\section{Guidance for clinicians in primary care and hospital general medicine}

GO commonly presents with symptoms of ocular irritation, light sensitivity or watering in the context of new swelling or redness of the eyelids or eyes (Box 1, S1). Note that proptosis may not be present. The majority of patients newly presenting with GO have disturbance of thyroid function, usually hyperthyroidism. The association between smoking and GO is strong and consistent. ${ }^{7}$ Selenium supplements in the form of sodium selenite at a dose of $100 \mu \mathrm{g}$ twice daily (equivalent to $91.3 \mu \mathrm{g}$ of elemental selenium twice daily), given for 6 months, are beneficial in patients with active mild GO. ${ }^{8}$ Thyroid dysfunction is detrimental to GO. ${ }^{9}$ Artificial tears can alleviate symptoms of corneal exposure (usually causing grittiness, photophobia and excessive watering) and prevent corneal ulceration. ${ }^{9}$ Diplopia is one of the most distressing symptoms of GO. Prisms may provide immediate symptomatic relief and can be adjusted as necessary during the changeable phases of GO.

Table 1 defines key tiers of care and outlines the roles of healthcare professionals involved in the management of patients with GO.

\section{Recommendations}

i. Consider the diagnosis of GO in patients being treated for 'conjunctivitis' or 'ocular allergy' when symptoms are persistent and fail to respond to treatment.

ii. Assess clinically, based on history and examination, the likelihood of GO in patients presenting with 'conjunctivitis' or 'ocular allergy' (Box 1).

iii. Order thyroid function tests in all patients newly diagnosed or suspected to have GO.

iv. Offer smoking cessation advice and referral to smoking cessation services to all patients known or suspected of having Graves' disease or GO who are current smokers $(2+, B)$.

v. Provide good quality information on the effects of smoking and GO to patients who are current smokers. Disease-specific patient information is available via the British Thyroid Foundation. ${ }^{10} \square$

vi. Advise patients with a new diagnosis of mild GO to commence on a course of sodium selenite at a dose of 100 $\mu \mathrm{g}$ twice daily (equivalent to $91.3 \mu \mathrm{g}$ of elemental selenium twice daily) for 6 months $(1+, \mathrm{A})$.

vii. Correct hypothyroidism immediately with either introduction or adjustment of the dose of levothyroxine in accordance with national guidelines ${ }^{11}(2+, \mathrm{C})$.

viii. Render patients with newly diagnosed GO and biochemical evidence of hyperthyroidism euthyroid as quickly as possible either by initiation of anti-thyroid drug treatment and careful monitoring to avoid hypothyroidism (as agreed by local protocols) or by immediate referral to tier 2 (Table 1, Fig 1) $(2+, \mathrm{C})$.

ix. Advise all patients with GO and symptoms suggestive of corneal exposure to use lubricant eye drops.

$\mathrm{x}$. Consider prismatic correction (through referral to an orthoptist) in all patients with GO who have troublesome diplopia.

xi. Refer patients whose dominant clinical problem is hyperthyroidism to tier 2 (Table 1, Fig 1).

xii. Refer patients whose dominant clinical features are due to GO directly to tier 3 (Table 1, Fig 1), and if hyperthyroid also to tier 2, unless tier 3 is a joint thyroid eye clinic.

xiii. Refer patients with an unclear ophthalmological diagnosis to tier 3 (Table 1, Fig 1).

\section{Guidance for endocrinologists and nuclear medicine physicians}

Many patients with GO develop eye disease after the diagnosis of Graves' disease and during follow up in thyroid clinics. All patients with Graves' disease at the time of diagnosis of hyperthyroidism are at risk of developing GO particularly if smokers. Algorithmic diagnostic criteria for GO (S2) and classifications of disease activity and severity (S3) are recognised (although the latter require detailed ophthalmological assessments normally beyond the scope of most endocrinologists and nuclear medicine physicians). A simple clinical assessment tool can identify patients in thyroid clinics who may benefit from referral to a tertiary centre (S4). ${ }^{12}$ Awareness of the early symptoms of GO (Box 1) by patients with Graves' disease using an early warning card (S5), may facilitate early diagnosis. Radioiodine may cause 


\section{Table 1. Tiers and roles of services for patients with GO.}

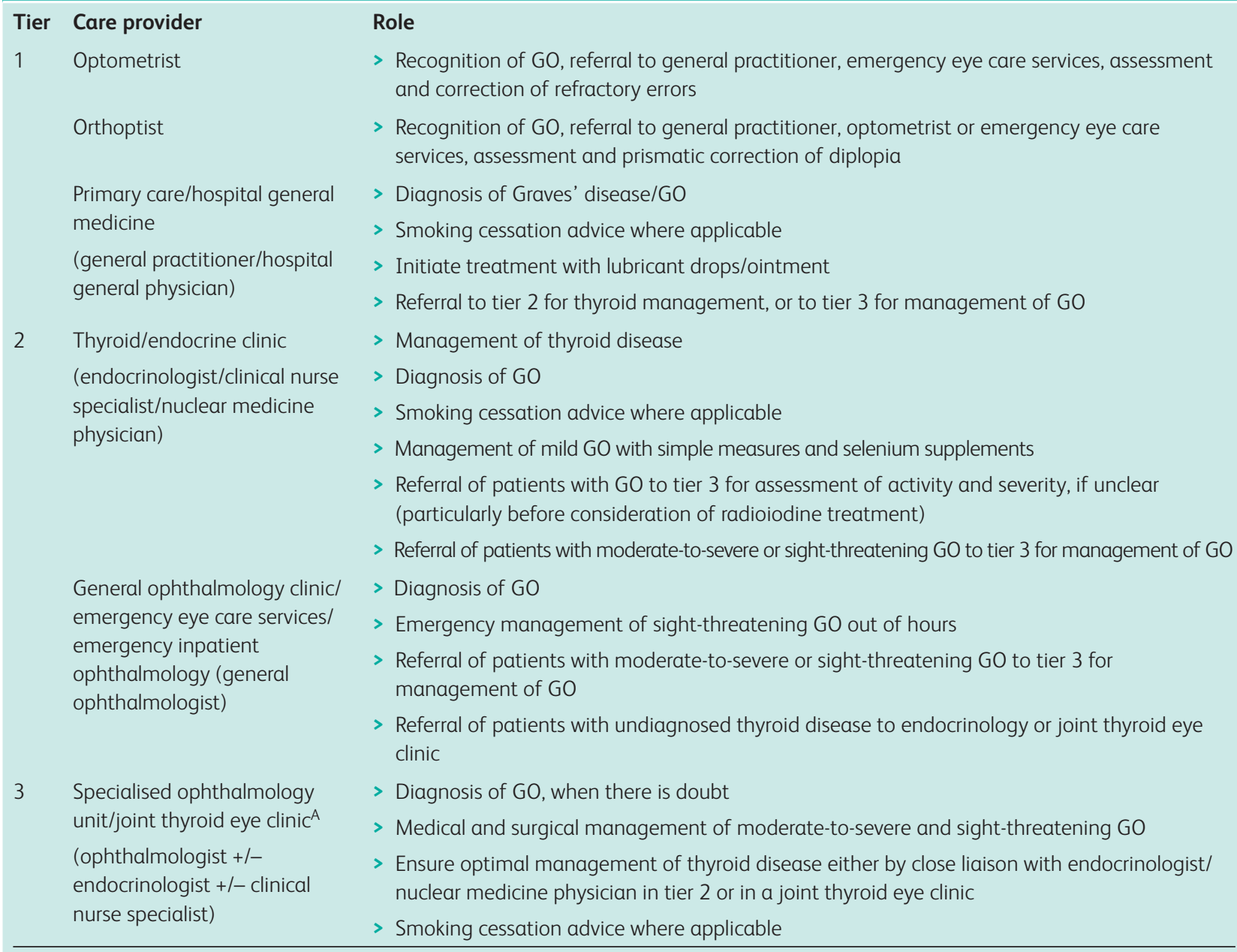

Ajoint thyroid eye clinics are formally joined up clinics with endocrinologists and can offer an integrated service both for the eyes and the thyroid. GO = Graves' orbitopathy.

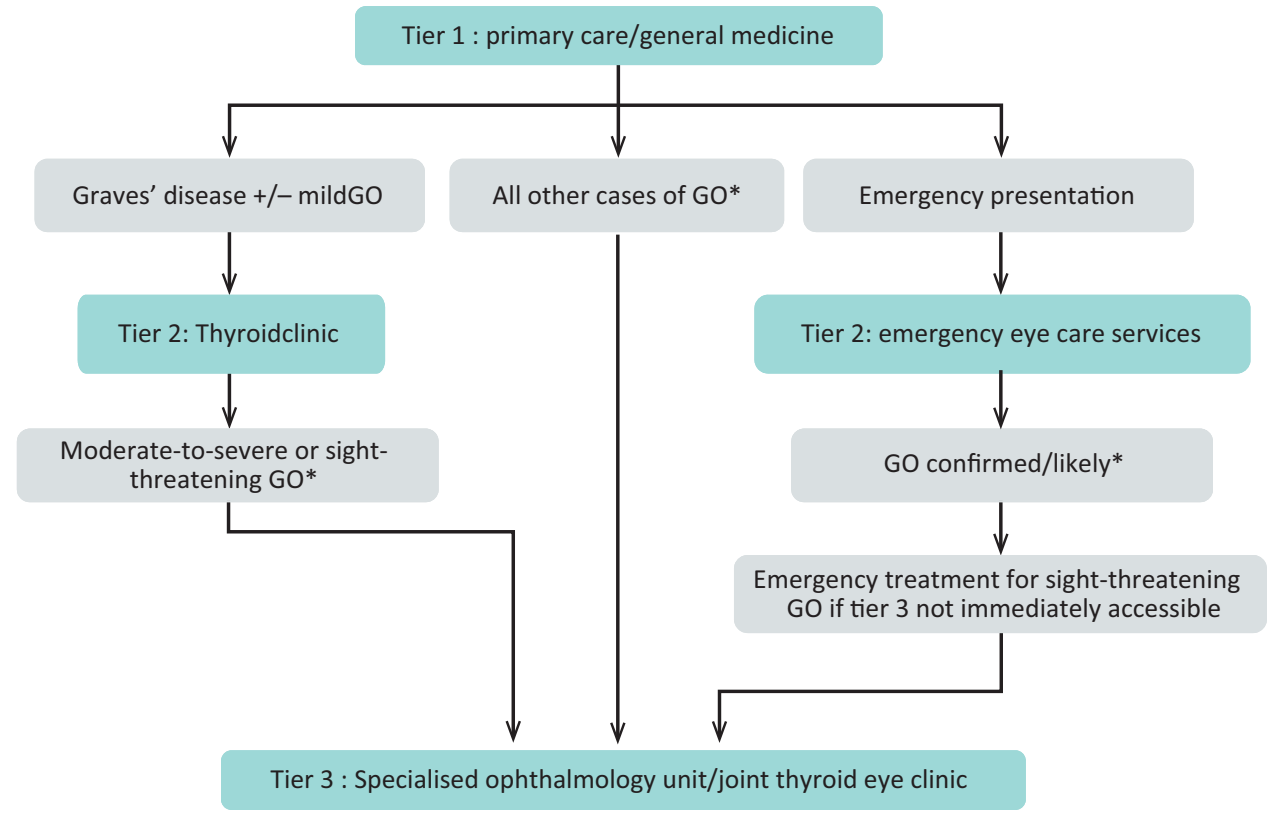

Fig 1. Referral pathways for patients with GO. *Patients with blurred or double vision should also be referred to orthoptic service for consideration of prismatic correction. $\mathrm{GO}=$ Graves' orbitopathy. 
exacerbation of GO. ${ }^{9}$ If radioiodine is necessary, a course of prophylactic steroids may be indicated unless the GO is inactive, when radioiodine can be administered without steroid prophylaxis. ${ }^{9}$

\section{Recommendations}

i. Make an accurate diagnosis of Graves' disease in all patients presenting with thyrotoxicosis, as this will identify those at risk of GO. $\square$

ii. Counsel all patients diagnosed with Graves' disease who are smokers about smoking cessation and offer disease-specific information ${ }^{10}$ and smoking cessation interventions. $\square$

iii. Give written information about the early symptoms of GO (S5) to all patients diagnosed with Graves' disease. $\square$

iv. Screen patients with Graves' disease attending thyroid clinics for GO using a brief focused clinical assessment tool (S4).

v. Initiate interventions described under 'Guidance for clinicians in primary care and hospital general medicine' above (recommendations iv, $v$, vi, ix and $\mathrm{x}$ ) if appropriate.

vi. Achieve and maintain euthyroidism as soon as possible $(2+, \mathrm{C})$.

vii. Avoid radioiodine unless the eye disease is inactive, as confirmed by an ophthalmologist experienced in GO. Otherwise, cover with a course of oral steroids, using prednisone $0.3-0.5 \mathrm{mg} / \mathrm{kg} 1-3$ days after radioiodine and slowly reducing over 3 months $(1+, \mathrm{A})$.

viii. Take particular care to avoid hypothyroidism when radioiodine is used. Measure thyroid function every 1-2 months for the first 6 months beginning no later than 4 weeks post-radioiodine. Introduce thyroxine when free T4 levels are low or showing a downward trend within the normal reference range, even in the absence of a raised TSH, as the rise in TSH may be delayed. $\square$

ix. Patients with inactive GO can be treated with radioiodine without steroid cover, provided hypothyroidism is avoided $(2+, \mathrm{C})$.

\section{Guidance for general ophthalmologists}

General ophthalmologists may need to treat emergency complications if tier 3 services are not immediately accessible, but their main role is to ensure that such cases are referred to tier 3, and to an endocrinologist or nuclear medicine physician when thyroid dysfunction is identified (unless the tier 3 service offers a joint thyroid eye clinic).

\section{Recommendations}

i. Order thyroid function tests in all patients newly diagnosed or suspected to have GO.

ii. Initiate immediate treatment for sight-threatening GO due to severe corneal breakdown and globe subluxation. $\square$

iii. Initiate treatment for sight-threatening GO due to severe dysthyroid optic neuropathy (DON) associated with significant sight loss if immediate transfer of care to tier 3 is impractical. $\square$
1. Has your vision changed in either eye-despite wearing up-to-date spectacles, contact lenses or reading glasses?

2. Do you have any areas of blurring in your field of vision that do not resolve on blinking or covering one or the other eye?

3. Do colours look different if you close each eye in turn?

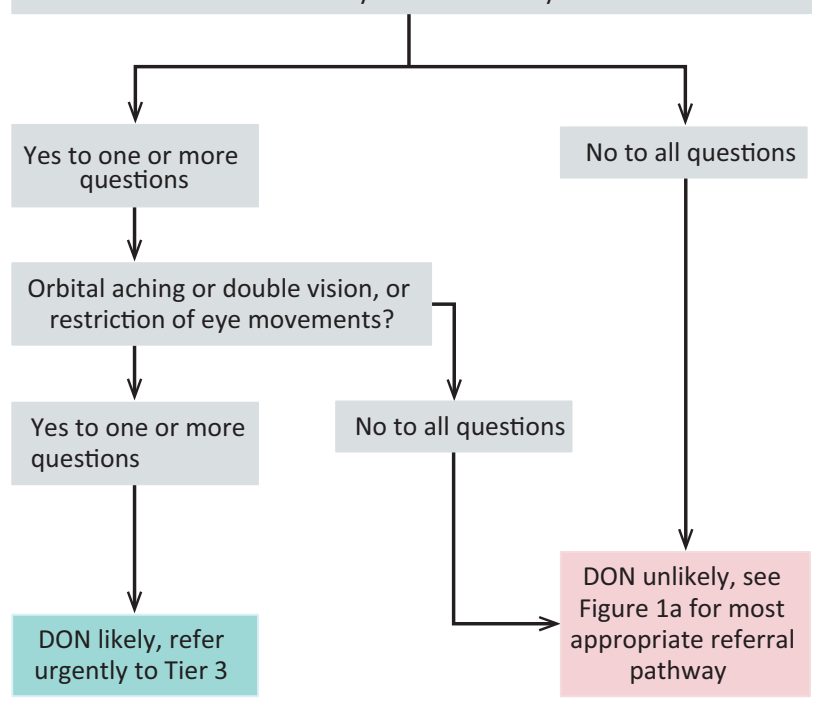

Fig 2. Algorithm for identification of DON. DON = dysthyroid optic neuropathy.

iv. Refer all other cases of GO routinely to tier 3 for further management (Fig 1) $\square$

v. Initiate interventions described under 'Guidance for clinicians in primary care and hospital general medicine' above (recommendations iv, $v, v i$, ix and $\mathrm{x}$ ), if appropriate.

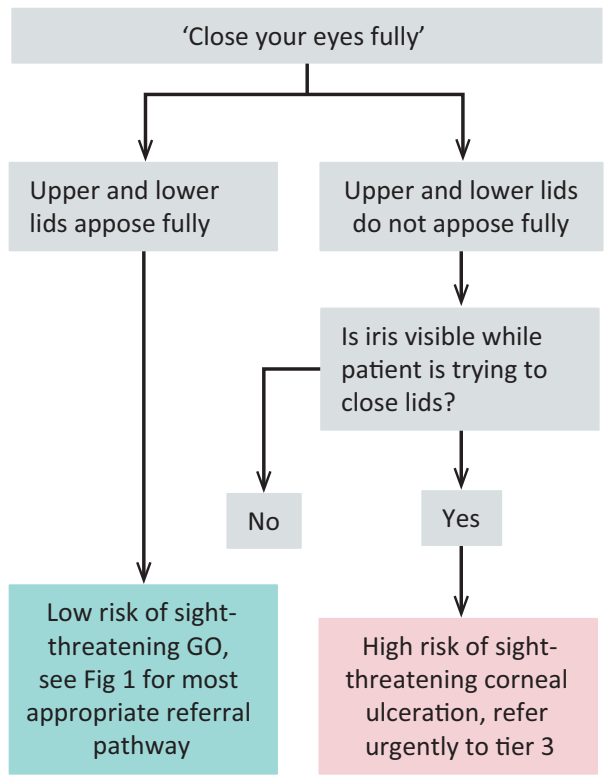

Fig 3. Algorithm for identification of patients with sight-threatening corneal ulceration. 


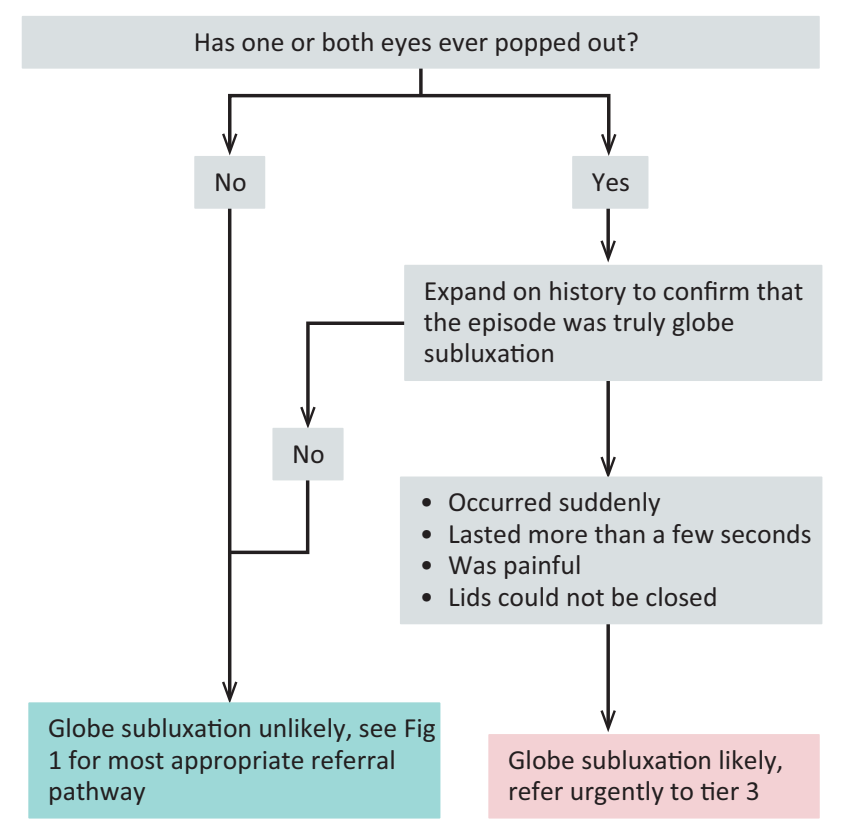

Fig 4. Algorithm for identification of patients with a history of globe subluxation.

\section{Recognition of sight-threatening GO}

Sight-threatening GO is rare and can be due to DON, corneal ulceration or globe subluxation. A structured step-wise assessment will help the non-specialist physician determine whether DON is likely, and will guide as to the need for urgent referral (Fig 2). 'Red flag' signs for corneal breakdown can be identified by performing a simple clinical assessment (Fig 3). The diagnosis of globe subluxation is obvious and patients usually present as an emergency. A history of globe subluxation can be elicited by asking patients appropriate questions (Fig 4).

\section{Recommendations}

i. If sight-threatening GO is suspected, use the algorithms in Figs 2-4 for clinical evaluation.

ii. Refer patients with sight-threatening GO urgently to a tier 3 centre (Fig 1).

iii. Refer ophthalmological emergencies occurring out of hours to the nearest emergency eye care services (Fig 1).

\section{Pathways of care between tiers for optimal} management of patients with GO

Optimal care for patients with GO requires efficient flow in well-defined pathways.

\section{Recommendation}

i. The pathways outlined in Fig 1 are recommended. $\square$

\section{Communication between tiers of care and patients with $\mathrm{GO}$}

Good communication between tiers of care and between health professionals and patients is paramount and can improve outcomes. Shared decision making can facilitate management and enhance the patient experience. ${ }^{13}$

\section{Recommendations}

i. Develop and maintain efficient and robust channels of communication between tiers of care and between health professionals and patients with GO. $\square$

ii. Consider shared decision making in management decisions for patients with GO. $\square$

\section{Patient support}

Patients with GO often are in need of moral and psychological support. In some cases formal psychological support may be helpful. Patient-led organisations, such as the British Thyroid Foundation (www.btf-thyroid.org) and the Thyroid Eye Disease Charitable Trust (www.TEDct.org.uk) have an important role in helping patients cope with their disease.

\section{Recommendations}

i. Offer psychological support to selected patients with GO who find it difficult to cope with their disease. $\square$

ii. Offer contact details of patient-led organisations to all patients with GO. $\square$

\section{Limitations of the guidance}

Many of the recommendations are based on descriptive studies and the experience and opinion of the guideline development group.

\section{Implications for implementation}

Implementation of this guidance may increase the number of referrals to specialised centres and the number of patients offered treatments. This is expected to be offset by better outcomes and less long-term morbidity. Standards for audit have been set by the Amsterdam Declaration for Graves' Orbitopathy. ${ }^{4}$

\section{Acknowledgements}

The authors wish to thank Miss Tamsin Sleep (consultant ophthalmologist and clinical director, South Devon Healthcare NHS Trust), Professor Bernard YP Chang (consultant ophthalmologist and lead clinician, Leeds Teaching Hospitals NHS Trust; vice president, the Royal College of Ophthalmologists), Ms Rowena McNamara (chair, British and Irish Orthoptic Society), Dr Sian Roberts (general practitioner and TED patient) and Mr David Parkins (president, the College of Optometrists) and Dr Susan Blakeney (clinical adviser, the College of Optometrists) for their helpful comments on the manuscript.

\section{References}

1 Kahaly GJ, Petrak F, Hardt J, Pitz S, Egle UT. Psychosocial morbidity of Graves' orbitopathy. Clin Endocrinol (Oxf) 2005;63:395-402.

2 MacEwen C. Know how to diagnose and manage thyroid eye disease. GPonline, 14 March 2012. Available online at www.gponline.com/ Clinical/article/1120742/expert-opinion-diagnosing-thyroid-eyedisease/ [Accessed 3 February 2015].

3 Perros P, Chandler T, Dayan CM et al. Orbital decompression for Graves' orbitopathy in England. Eye 2012;26:434-7. 
4 Perros P, Wiersinga WM. The Amsterdam Declaration on Graves orbitopathy. Thyroid 2010;20:245-6.

5 Thyroid Eye disease Amsterdam Declaration Implementation Group UK. TEAMeD. Available online at www.btf-thyroid.org/ index.php/campaigns/teamed [Accessed 3 February 2015].

6 Scottish Intercollegiate Guideline Network. SIGN 50. A guideline developer's handbook (2014). Edinburgh: SIGN, 2014. Available online at www.sign.ac.uk/pdf/sign50.pdf [Accessed 3 February 2015]

7 Wiersinga WM. Smoking and thyroid. Clin Endocrinol (Oxf) 2013;79:145-51.

8 Marcocci C, Kahaly GJ, Krassas GE et al. Selenium and the course of mild Graves' orbitopathy. N Engl J Med 2011;364:1920-31.

9 Bartalena L, Baldeschi L, Dickinson A et al. Consensus statement of the European Group on Graves' orbitopathy (EUGOGO) on management of GO. Eur J Endocrinol 2008;158:273-85.

10 British Thyroid Foundation. Graves' disease, thyroid eye disease and smoking: information for patients (2014). Available online at www. btf-thyroid.org/index.php/thyroid/articles/23-general-btf-articles/ our-campaigns/431-ted-smoking-feedback [Accessed 3 February 2015].

11 Royal College of Physicians. The Diagnosis and Management of Primary Hypothyroidism. London: RCP, 2008. Available online at www.rcplondon.ac.uk/resources/clinical-resources/diagnosis-andmanagement-primary-hypothyroidism [Accessed 3 February 2015].

12 Mitchell AL, Goss L, Mathiopoulou L et al. On behalf of TEAMeD-UK (Thyroid Eye Disease Amsterdam Declaration Implementation Group UK). DiaGO (Diagnosis of Graves' Orbitopathy): results of a pilot study to assess the utility of an office tool for practising endocrinologists. J Clin Endocrinol Metab 2014;Dec 8:jc20143146.

13 Elwyn G, Coulter A, Laitner S et al. Implementing shared decision making in the NHS. Brit Med J 2010;341:c5146.

Address for correspondence: Dr P Perros, Department of Endocrinology, Elliott Building, Royal Victoria Infirmary, Newcastle upon Tyne NE1 4LP, UK.

Email: petros.perros@ncl.ac.uk

\section{Gifts and memorabilia}

A new range of gifts based on a huge collection of art, and artifacts housed at the RCP's home in Regent's Park.

Elizabeth Garrett Anderson bag Large jute bag reinforced with a synthetic lining and short padded handles.

$£ 10.00$ (inc p+p)

Royal College of Physicians

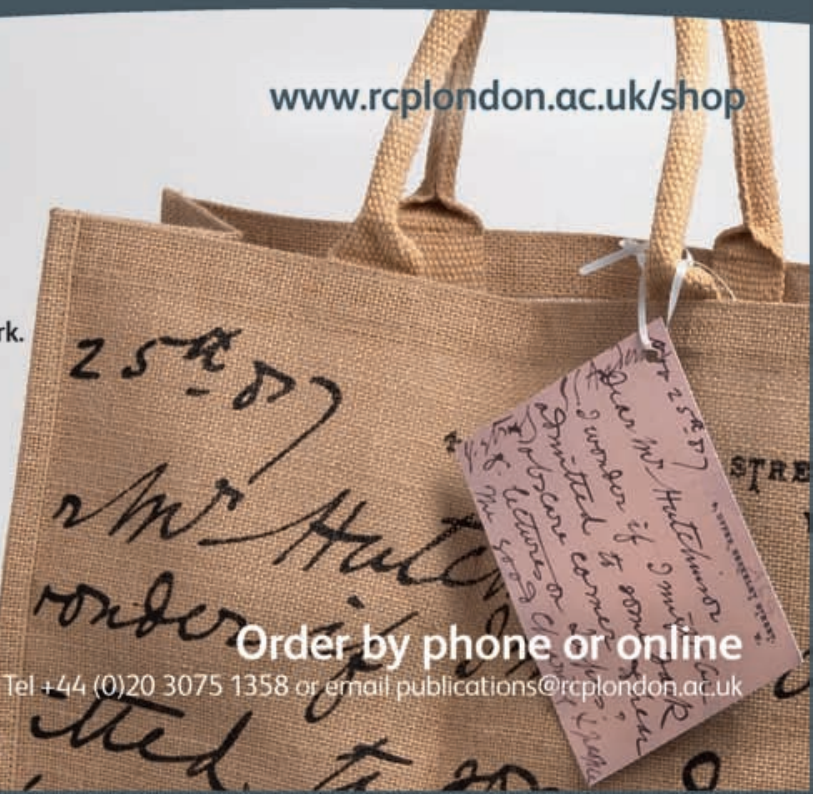

